\title{
Diskussion zum Vortrag von Christiane Wendehorst
}

\author{
Leitung: Uwe Diederichsen
}

DiEDERICHSEN:

Vielen Dank für Ihr Referat, das sehr viele Einblicke gewährte, aber vielleicht doch den einzigen Nachteil hatte, dass es so klar war, dass nicht deutlich wird, wie kompliziert und unverständlich diese europäischen Regelungen sich in unserem BGB zum Teil ausmachen. Sie haben sich im Rahmen der Zeit gehalten, eine halbe Stunde war für die Diskussion vorgesehen und wir können uns dies ohne Weiteres leisten. Herr Link als Erster.

LiNk:

Frau Wendehorst, das war ein schöner Vortrag, der mich aber auch durch seine Analysen erschreckt hat. Was mir dabei aber nicht ganz klar geworden ist - dies mein erster Punkt -, ist die Abgrenzung zum Paternalismus. Geht es beim Verbraucherschutz nicht auch gerade darum, den Verbraucher paternalistisch zu schützen, auch wenn er diesen Schutz gar nicht will oder nicht benötigt? Ist das wirklich edukatorisch? Der zweite Punkt ist mehr ein allgemeiner: Wir beobachten doch im Grunde seit vielen Jahren eine Moralisierung des Privatrechts, nicht zuletzt durch die von der Judikatur immer mehr verfeinerte Drittwirkung der Grundrechte und nun auch - teils europäisch, teils grundrechtlich begründet - etwa durch die Antidiskriminierungsgesetzgebung, die für mich jedenfalls einen Fremdkörper in der privatrechtlichen Dogmatik darstellt und die mit dem rein edukativen Impetus auftritt, Privatrecht oder Privatrechtsgebrauch moralisch aufzurüsten. Damit - so scheint mir - werden alte, über 2000 Jahre erprobte Regelungsmechanismen des Privatrechts, die eher auf einen Interessenausgleich zielen, aufgeweicht oder teilweise gar über Bord geworfen.

WENDEHORST:

Gut, zunächst zur Abgrenzung zwischen Paternalismus und edukatorischem Charakter. Nach meiner Begriffsdefinition ist der Übergang ohnehin fließend, d.h. wir haben bei fast jeder Regelung vermutlich irgendwie, wenn auch ganz, ganz schwach ausgeprägt, ein paternalistisches und möglicherweise edukatorisches Element. Das ist alles eine Frage der Schwerpunktsetzung. Und ich würde Ihnen auch Recht geben, dass bei den frühen verbrauchervertraglichen Richtlinien das paternalistische Element deutlich überwiegt. Das ist meines Erachtens kaum bestreitbar. Jetzt in jüngerer Zeit sehe ich dagegen die Tendenz, dass es plakativ ausgedrückt oder überspitzt formuliert - dem europäischen Gesetzgeber 
fast schon gleichgültig ist, welche Schutzinstrumente im Gesetz stehen. So ist es zum Beispiel interessant, dass, wenn man sich bemüht, das Widerrufsrecht bei Kreditverträgen auf seine Berechtigung hin zu überprüfen, man kaum Aussagen des europäischen Gesetzgebers findet, warum er das in die Richtlinie - noch dazu vollharmonisierend für ganz Europa - hineingeschrieben hat. Da steht nur: Das war auch schon so in der Richtlinie über den Fernabsatz und Finanzdienstleistungen, das haben wir jetzt einfach wieder gemacht. Und wenn man die europäischen Dokumente analysiert und auch die Diskussionen verfolgt, so gewinnt man den Eindruck, dass das Hauptziel ist, hier einen einheitlichen Binnenmarkt zu schaffen, und dass sozusagen die eigentlichen Schutzinstrumente, in denen sich der paternalistische Charakter in Bezug auf den Verbraucher ausdrückt, von der Intention des europäischen Gesetzgebers her fast in den Hintergrund rücken.

Liberalisierung des Privatrechts, Drittwirkung der Grundrechte, Diskriminierung - ich denke das auch. Vor ungefähr einem Jahr ist Eva Schumann auf mich zugekommen und hat mich gefragt, ob ich über Diskriminierung oder Verbrauchervertragsrecht sprechen wolle. Ich habe ganz spontan, einfach weil ich gerade in dem Bereich gearbeitet hatte, gesagt: Dann nehme ich das Verbrauchervertragsrecht. Als ich den Vortrag schrieb, habe ich meine Wahl verflucht, weil ich so wenig fand, das wirklich meinem Begriffsverständnis von „edukatorisch“ entspricht, und dachte: „Hättest du doch die Diskriminierung genommen!“ Denn ich glaube auch, dass man dort deutlich mehr finden kann.

\section{DieDERICHSEN:}

Herr Behrends bitte.

\section{BEHRENDS:}

Eine weitere Frage, ob hinter oder neben dem oder vielleicht sogar vor dem Edukatorischen nicht die Zielsetzung liegt, für den europäischen Konsumenten eine einheitliche Umwelt und einheitliche Bedingungen zu schaffen. Gemeinsame Währung, gleiche Maße, gleiche Gewichte, gleiche DIN-Normen haben nichts Edukatorisches, sondern erzeugen durch die Einheitlichkeit, in der ihre jeweiligen Zwecke verfolgt werden, ein europäisches Vertrautheitsgefühl. Eine andere Frage ist, dass die Behauptung der Schutzbedürftigkeit oft nicht recht überzeugt. Denn unabhängig davon, ob ich ein verpacktes Industrieprodukt in einem Laden kaufe oder mir schicken lasse, meine Ignoranz, wie das Ding funktioniert, ist die gleiche. Da gibt es keinen Unterschied. Bei zu viel Schutz besteht jedenfalls die Gefahr, dass eine gewisse Sorglosigkeit gefördert wird, zumal da man gelegentlich auch den Eindruck hat, dass es bei der Formulierung von Schutzbedürfnissen auch um die Rechtfertigung von Eingriffsbefugnissen geht. Eine Bürokratie muss schließlich ihre Existenz rechtfertigen. Nach allem ist es 
nicht auszuschließen, dass es zu Erziehungswirkungen kommen kann, die ins Negative gerichtet sind. Wir empfinden als Erziehung, wenn jemand zur Selbständigkeit, zur Autonomie erzogen wird, und weiß, wenn er A sagt, dann muss er auch B sagen und also die Folgen seines Handelns tragen. Es kann aber, wenn zur Sorglosigkeit erzogen wird, das genaue Gegenteil bewirkt werden.

WENDEHORST:

Ja, also ich kann eigentlich bei allen drei Punkten nur zustimmend reagieren. Das mit der gewissen Vertrautheit in Europa denke ich, ist ein ganz starkes Element, war es allerdings meines Erachtens auch schon bei den alten Richtlinien. Das habe ich an einer Stelle meines Vortrags, glaube ich, erwähnt, dass man dem Verbraucher, sei es zu Hause oder sei es auf Reisen, quasi immer das gleiche rechtliche Umfeld, zumindest einen Mindeststandard dieses Umfeldes garantieren möchte. Das ist sicherlich ein ganz wichtiger Punkt. Er ist nur jetzt in der Rhetorik und in den Rechtfertigungen in der letzten Zeit in den Hintergrund getreten. Wir haben ein ähnliches Problem, wie wir das beim letzten Referat von Herrn Schroeder hatten, dass wir natürlich in gewisser Weise auch auf die Materialien zurückgreifen und schauen müssen, wie wird dieser konkrete Rechtsakt, diese konkrete Maßnahme gerechtfertigt. Und da sehen wir jetzt z.B. bei den ganzen Diskussionen um die Verbraucherrechterichtlinie, dass fast nur noch argumentiert wurde mit dem Unternehmer, den kleinen und mittleren Unternehmen, denen man es ermöglichen muss, irgendwie grenzüberschreitend in 27 Mitgliedsstaaten anzubieten. Das stand einfach schlichtweg für KunEwA oder Аввамоnte und wie sie alle heißen im Vordergrund. Das Andere ist aber immer auch noch da; es ist wie beim Paternalismus, insofern gebe ich Ihnen auch vollkommen Recht.

Und Erziehung ins Negative, selbstverständlich: Ich bin z.B. der Überzeugung, dass das Widerrufsrecht für Kreditverträge völlig kontraproduktiv ist. So als würde man Informationen, wenn man den Vertrag unterschreibt, genauer lesen als vorher: Das ist überhaupt nicht der Fall. Es gibt im Gegenteil Untersuchungen, dass das genau umgekehrt ist.

\section{DIEDERICHSEN:}

Frau Langenfeld, Sie haben das Wort.

\section{LANGENFELD:}

Erstens eine Frage: Wie sind Deine Erkenntnisse darüber, wer diese Verbraucherschutzregulierungen oder die Regulierung des Privatrechts im Verbraucherschutzbereich stark befördert? Ist das nicht auch die Wirtschaft, die natürlich ein Interesse daran hat, dass die Dinge vereinheitlicht werden und den Verbraucher ermutigen möchte, Geschäfte grenzüberschreitend verstärkt zu tätigen, auch 
wenn dadurch möglicherweise Verbraucherschutzrechte gleichzeitig verstärkt werden. Vielfach werden sie ja dann doch nicht in Anspruch genommen. Der zweite Punkt bezieht sich auf das Allgemeine Gleichbehandlungsgesetz, also das deutsche Umsetzungsgesetz zu den gemeinschaftlichen Antidiskriminierungsrichtlinien, die ja einen deutlich überschießenden Charakter haben, namentlich im allgemeinen zivilrechtlichen Bereich. Hier kann man nicht nur von einem edukatorischen, sondern von einem geradezu einschüchternden Effekt sprechen. Denn es wird vielfach Arbeitgebern, aber auch Vermietern geraten, Gespräche über den Abschluss eines Arbeitsvertrages oder den Abschluss eines Mietvertrages nur noch mit Zeugen zu führen und äußerste Zurückhaltung im Gespräch zu wahren. Der deutsche Gesetzgeber hat sich hier mit dem europäischen Gesetzgeber im Verbund daran gemacht, Moral und Recht zu verbinden, in einer, wie ich finde, sehr unangenehmen Weise. Und das dritte, und das ist besorgniserregend und führt vielleicht über das eigentliche Tagungsthema hinaus, ist, in welcher Weise das europäische Recht Fremdkörper in das nationale Recht hineinimplantiert. Das ist nicht nur im Privatrecht so, sondern auch in weiten Teilen des Öffentlichen Rechts, wenn ich etwa an das Umweltrecht denke oder andere Rechtsgebiete. Ich darf das für das Antidiskriminierungsrecht präzisieren: Allein die Beweislastregelungen, die Regelungen über die Sanktionen, die Regelungen zur Altersdiskriminierung etwa im Versicherungsbereich, sind vom deutschen Recht nur schwer zu bewältigen. Das sind Fremdkörper und das ist eine enorme Herausforderung für die Gerichtsbarkeit, aber auch für die Kommentatoren. Wie siehst Du diese Entwicklung? Kann man da vielleicht noch auf eine Veränderung des Bewusstseins dahingehend hoffen, dass man stärker sieht, dass solche Rechtsnormen des europäischen Rechts in die nationalen Rechtsordnungen überführt werden müssen und dort in den Rahmen dessen, was das nationale Recht aus gutem Grunde über viele Jahre entwickelt hat, hineinpassen müssen?

\section{WENDEHORST:}

Ich werde versuchen, die Fragen der Reihe nach kurz zu beantworten. Erstens, wer befördert das: Ja, das ist eine schwierige Geschichte. Vordergründig wohl in der Tat die Wirtschaft. Und es ist wohl auch so, wie Du sagst, dass es der Wirtschaft eigentlich ganz egal ist, wie gut das Widerrufsrecht ausgestaltet wird, denn es widerrufen ohnehin nur wenige Verbraucher, und diese paar Fälle kann man wirtschaftlich vernachlässigen. Es geht der Wirtschaft um Zugang zu Märkten. Wie schaffe ich den Zugang zu bestimmten Märkten, ohne dass ich durch Unterlassungsklagen vom Wettbewerb vertrieben werden kann? Allerdings ist das natürlich nur eine vordergründige Sichtweise. Ich denke, wenn man die Sache in der Mikroperspektive betrachtet, ist sie viel komplexer, und es liegt, wenn 
ich das einmal so ungeschützt sagen darf, auch vieles am individuellen persönlichen Ehrgeiz einzelner Kommissionsbeamter, verschiedener Mächte innerhalb der Kommission, wo sich jeder profilieren und ein wieder neueres und noch schöneres Instrument zur Beförderung des Binnenmarktes präsentieren möchte. Ich habe das Gefühl, dass die Erklärung für manches viel profaner ist, als es uns erscheint.

Zur Frage nach dem überschießenden Charakter des Allgemeinen Gleichbehandlungsgesetzes: Ich denke auch, dass man hier deutlich über das Erforderliche hinausgegangen ist, und zwar mit bedenklichen Folgen. Das kann ich eigentlich im Prinzip nur zustimmend kommentieren.

Fremdkörper im nationalen Recht: Ja, das habe ich versucht zu zeigen. Das Bewusstsein dafür sehe ich allerdings noch relativ schwach ausgeprägt. Es ist natürlich auch schwierig: Es gibt ja nicht nur die deutsche Rechtskultur, sondern es gibt viele Rechtskulturen. Wenn man die Europäisierung will, wenn man auch Rechtsintegration in Europa will, kann man natürlich nicht unbedingt den Anspruch erheben, dass alles gerade in die deutsche Dogmatik hineinpasst. Eine gewisse Zurückhaltung wäre die Konsequenz des Ganzen. Den Bewusstseinswandel sehe ich in diesem Zusammenhang noch nicht so klar.

\section{DIEDERICHSEN:}

Ich habe jetzt auf der Liste Herrn Wißmann, Herrn Stolleis, Herrn Rückert, Herrn Marburger, Herrn Simon und Herrn Zimmermann. Und wir haben noch 20 Minuten, aber ich glaube, wenn wir fünf Minuten dranhängen, dann kommen wir durch, wenn Sie sich kurz fassen und sich auf wenige Fragen beschränken. Herr Wißmann bitte.

\section{WISSMANN:}

Vielen Dank. Ich will im Anschluss an Frau Langenfeld auch erst einmal betonen, wie gut geeignet dieses Thema für einen intradisziplinären Dialog ist. Das ist sozusagen schon an Begriffen festzumachen. Es gibt eben nicht nur eine Regulierung des Privatrechts, sondern es gibt auch eine Regulierung des Verwaltungsrechts und das kümmert sich, der Zufall will es, auch um Verbraucherrechte. Aus unserer öffentlich-rechtlichen Sicht geht es dabei zunächst vielfach um die Rolle des Staates - in der Wirtschaftsaufsicht, in der Infrastrukturregulierung -, aber dahinterliegend um die Rolle des Bürgers: Was für eine Vorstellung vom Bürger haben wir eigentlich, wie ist er in der Lage, Verträge abzuschließen, Herr seiner selbst zu sein, und wo ist er das nicht? Ich hätte jetzt zwei knappe Fragen oder Anmerkungen. Erstens, aus meiner Sicht ist der Kern des Verbrauchervertragsrechts doch nach wie vor, auch als neue Herausforderung für das deutsche Recht, der zwingende Charakter bestimmter Regelungen. Sie haben das ja auf dem neuesten Stand und auch in einer weiteren Perspektive geschildert, aber 
das scheint mir doch nach wie vor der Kern zu sein. Und ist das nicht für das Zivilrecht auch wirklich eine ungeheure Herausforderung, weil es legitimatorisches Neuland bedeutet. Bisher konnte man sagen, Zivilrecht dient der Senkung von Transaktionskosten; der Gesetzgeber macht allgemeine Regeln, man kann sich aber auch anders einigen. Wenn jetzt ein Gesetz sagt: So ist es richtig und darauf einigt ihr euch oder ihr einigt euch gar nicht, dann muss das BGB sich gegenüber den Bürgern neu legitimieren, weil es ihnen eben nicht mehr nur hilft, jedenfalls einer Seite nicht. Der kontraintuitive Effekt in Bezug auf unser Thema heute ist doch aber vielleicht, dass Erziehung hier letztlich doch nur viel oberflächlicher und direkter stattfindet als bisher, aber nicht notwendig mit größerem Anspruch. Denn auch das dispositive Recht will doch letztlich die Erziehung hin zu Leitbildern: „Was ist ein vernünftiger Vertrag?“ - im Normalfall sollte man sich daran halten. Das ist doch auch ein Erziehungsanspruch, von dem man freilich abrücken kann. Der Anspruch der Ausrichtung auf solche Vertragsverhältnisse war und ist aber in gewisser Weise sogar weitergehend als wenn man einfach die Leute dazu zwingt, es so zu machen. Das wäre die erste Frage. Und die zweite Frage: Wie gewinnt denn der Staat, der europäische Mehrebenenstaat, seine Erziehungsziele? Wir haben jetzt viel von politischen oder zufälligen Einflüssen gehört. Die Frage an Sie, aber vielleicht auch in die Runde wäre: Was trägt denn die Verfassung hier aus? Da frage ich Sie vielleicht ein bisschen überfallmäßig, aber das ist eine Frage, die uns natürlich besonders umtreibt. Wie stehen denn Gesetz und Verfassung zueinander? Wir reden hier vom erziehenden Gesetz. Was ist denn mit der erziehenden Verfassung?

\section{RÜCKERT:}

Die Verfassung ist auch ein Gesetz.

\section{WENDEHORST:}

Also, zunächst einmal zur Herausforderung durch den zwingenden Charakter: $\mathrm{Ja}$, ich halte das für eine Herausforderung, aber für keine so große. Das ist eine Entwicklung, die wir schon seit Jahrzehnten mitmachen, dass wir immer mehr distributive Elemente in das Privatrecht aufnehmen, da gehen teilweise auch zwingende Regelungen mit einher. Dies ist ein gewisser Fremdkörper, aber ich glaube, einer, den das Privatrecht inzwischen gelernt hat, zu bewältigen und mit ihm umzugehen. Während es noch nicht gelernt hat, mit diesen ganz neuen Einflüssen durch das, was ich „Regulierungsprivatrecht" genannt habe, wirklich umzugehen.

Darin, dass Erziehung hier direkter und oberflächlicher stattfindet als durch das klassische dispositive Privatrecht, gebe ich Ihnen absolut Recht. Das hat ein bisschen auch zu tun mit der Paternalismusdebatte, die wir geführt haben: 
Zwingendes Recht ist von vornherein paternalistischer in seinem Charakter, und damit, kann man auch sagen, verhindert es Erziehung.

Die Verfassungsdebatte ist natürlich eine, die wir wahrscheinlich morgen noch verstärkt führen werden. Man wird auch bei der Verfassung natürlich zunächst einmal ähnliche Maßstäbe anlegen können, d.h. fragen können, welche Verfassungsnormen in welchem Maße edukatorischen Charakter haben. Ich denke, dass man hier ganz stark fündig werden wird, möglicherweise sogar in viel größerem Maße fündig werden wird als im einfachen Gesetz.

Wie gewinnt der Staat seine Erziehungsziele? Das ist eine gute Frage, die haben wir heute Morgen, glaube ich, schon angeschnitten. Ich denke, das ist eine so große Frage, die würde ich ganz gern auf die Schlussdiskussion verschieben, weil ich sie in diesem Rahmen nicht bewältigen kann.

StOLleis:

Frau Wendehorst, Sie haben am Anfang analytisch klar ,edukatorisch“ und ,paternalistisch" getrennt, haben aber dann in der Diskussion gesagt, die Grenzen verschwimmen. Und ich glaube, das ist auch richtig so, denn der pater, der erziehen will, macht das ja auch nicht nur durch Repression, sondern auch durch Edukation; denn er möchte ein besser erzogenes, freiwillig den Geboten folgendes Kind haben. Und in diesem Sinn ist edukatorisch immer auch paternalistisch. Ich glaube, wir sind uns auch einig, besonders in Bezug auf den letzten Teil Ihres Vortrags über die Diagnose, die Sie gestellt haben, nämlich das zunehmende Verschwimmen der Grenzen zwischen öffentlichem und privatem Recht und Strafrecht. Charakteristisch ist eine relative Gleichgültigkeit gegenüber dem Einsatz des Mittels, wenn nur das Ziel erreicht wird. Und wenn die Ziele so im Vordergrund stehen, dann muss man doch fragen, so ähnlich wie Herr Wißmann: Welches Menschenbild hat ein heutiger EU-Kommissar? Und ich will es einmal außerhalb des Vertragsrechts ganz kurz illustrieren. Im Lebensmittelrecht wird gesagt: Du sollst nicht fett, du sollst nicht zu salzig, du sollst nicht zu süß essen, und dafür werden Mittel eingesetzt. Und weiter: Du sollst im Straßenverkehr Helme tragen. Du sollst energiesparende Lampen verwenden, du sollst den Müll trennen usw. Es gibt sozusagen ein Menschenbild der EU, vorangetrieben aufgrund ehrgeiziger Kommissare und dafür werden steuerrechtliche, strafrechtliche Mittel, Bußgeldmittel und das Zivilrecht instrumentell eingesetzt. Und diese Tendenz hat sich offenbar verstärkt. Christoph Link hat das „Ethisierung des Privatrechts“ genannt. Aber dann muss man doch selbstkritisch sagen, auch in der Rechtsprechung des Bundesverfassungsgerichts der Nachkriegszeit seit dem LÜTH-Urteil ist diese Ethisierung mit Hilfe der Grundwerte und der Wertordnungsjurisprudenz ganz bewusst und mit unser aller Zustimmung durchgesetzt worden. Wir wollten das Privatrecht und alle anderen Rechtsgebiete im 
Lichte unserer neuen Überzeugungen nach 1945 ausgestalten. Und das war im Grunde schon eine Ethisierung aller Rechtsgebiete. Das ist schrittweise gemacht worden und ist gelungen und wir haben das alle gebilligt. Jetzt erleben wir es auf europäischer Ebene. Ich sehe da durchaus eine Parallele.

\section{WENDEHORST:}

Zunächst einmal zur Bemerkung, dass Paternalismus und Erziehung verschwimmen: Ja, selbstverständlich, je genauer man hinsieht, desto weniger Konturen kann man ausmachen, desto weniger kann man die Grenze eigentlich ziehen. Da gebe ich Ihnen vollkommen Recht.

Jetzt zum Menschenbild eines EU-Kommissars: Ich fürchte, es ist jetzt vielleicht eine etwas bösartige Bemerkung, dass das Problem gerade darin liegt, dass der Mensch in dem Bild eigentlich ausgeblendet wird und viele Dinge eine Eigendynamik entwickeln. Sie haben angesprochen das Beispiel mit den Schutzhelmen. Ich meine, das ist ein typisches Beispiel, wo einfach Ideen wie Gefahrenprävention oder Gesundheitsfürsorge eine Eigendynamik entwickeln und der Mensch, der dem Ganzen ausgesetzt wird, eher nicht mehr vorkommt. Man hat ihn einfach überhaupt nicht im Auge, weil man sozusagen abstrakt Mechanismen konstruiert und durchspielt, die dann aber im Endeffekt für den Menschen eigentlich gar nicht geeignet sind. Ich habe - und auch das ist eine bösartige Behauptung - gelegentlich das Gefühl, dass der Mensch nur noch mit so abstrakten Termini umschrieben wird, wie „Akteur im Binnenmarkt“, „Nachfrager" usw., dass man sozusagen die Person hinter ihm eigentlich kaum noch erkennen kann.

Zur Frage der Ethisierung durch Verfassung und möglichen Parallelen: Ich würde sagen ja und nein. Eindeutig bildet sich die europäische Werteordnung auch durch Unrechtsvorstellungen auf europäischer Ebene heraus. Aber es ist doch auch das Demokratiedefizit, was wir hier möglicherweise auf europäischer Ebene haben. Die weiteren Wege der Rückkopplung sehe ich doch als zusätzliche Problematik bei dieser Ethisierung.

\section{DiEDERICHSEN:}

Ich würde jetzt sagen, wir fassen wieder zusammen wie bei den anderen Diskussionen auch. Frau Scheiwe, Herr Rückert und Herr Marburger.

\section{SCHEIWE:}

Vielen Dank für alles, was ich gelernt habe. Ich habe mich aber gewundert, nachdem Sie zu Anfang so genau differenziert haben, dass Sie dann bei dem Verbraucherkreditrecht vom edukatorischen Effekt par excellence gesprochen haben. Wenn man jetzt einmal zwischen Zielen und Effekten, also Wirkungen, trennt, dann kann man ja vielleicht von edukatorischen Zielen sprechen, aber mög- 
licherweise wird das auch wieder inflationär, weil dann alle normativen Ziele edukatorisch sind. Ich weiß nicht, ob das dann noch trennscharf ist. Aber wenn man dann von den Zielen, über die Sie ja eindrücklich gesprochen haben, auf die Effekte kommt, dann ist dies eine Vermischung. Es kann ein Ziel sein, das Bewusstsein von einem europäischen Verbraucher zu schaffen. Aber ob dieses Ziel erreicht wird und der Effekt erzielt wird, das wäre eine sozialwissenschaftliche Fragestellung. Darum habe ich mich gewundert, dass Sie von den edukatorischen Effekten gesprochen haben. Sollte man nicht die Ziele von den Effekten genauer trennen und das Normative nicht immer gleich als edukatorisch benennen?

\section{DIEDERICHSEN:}

Herr Rückert bitte, dann Herr Marburger.

\section{RÜCKERT:}

Ich will es ein bisschen zuspitzen, was ich jetzt alles gelernt habe. Und zwar, wenn Sie mit der Unterscheidung von Erziehung und Verhaltenssteuerung und Bewusstseinsbildung anfangen, dann ist das ja eine klassische Unterscheidung in der liberalen Theorie. Bei KANT ist ja der entscheidende Punkt, Innen- und AuBensteuerung zu unterscheiden, modern ausgedrückt. Das heißt, die analytische Unterscheidung ist sicher richtig und dass es empirisch dann manchmal nicht stimmt, das ist unerheblich für die Analytik. Nur finde ich es sehr interessant, dass herauskommt, dass die Unterscheidung eigentlich nicht so recht funktioniert. Dass es eigentlich der gesamten Privatrechtsgesetzgebung egal ist, ob das Recht innen oder außen steuert, weil es ankommt auf das objektive Ziel des Binnenmarkts. Und das finde ich dann einen ganz wichtigen Aspekt. Oder noch einmal in anderen Worten gesagt: Die ganze Erzieherei ist eigentlich irrelevant, man will ja nur den Binnenmarkt fördern. Das heißt, wir sind zurück bei der Unterscheidung, die Herr Simon gemacht hat: Es wird ein objektives Gut in den Mittelpunkt gestellt, ein kollektives Gut, und darum geht es und Erziehung ist sekundär. Wenn das stimmt, würde ich das noch mehr betonen, als Sie es getan haben. Diese Folgerung haben Sie noch nicht gezogen. Die zweite Geschichte ist - ein wichtiger Hinweis: Zwingendes Recht haben wir immer schon, damit können wir umgehen, aber jetzt kommt Regulierungsprivatrecht. Und da fragt man sich: Was ist eigentlich wirklich der Unterschied, das Neue? Sie haben es angedeutet mit dem Schlenker auf die Methodenlehre, das finde ich nun sehr interessant. Das hat mich veranlasst, sozusagen das Sprachspiel weiter zu spielen. Für die Methoden wäre die Konsequenz dessen, was Sie gesagt haben, Analogie ist vorbei, argumentum e contrario usw. ist vorbei. Das heißt, wir haben eigentlich ein Auslegungsverbot, ein Kommentierverbot und haben die Konsequenz des référé législatif, wie wir das um 1800 hatten bei dem Ideal der perfekten kasuisti- 
schen Befehlsgesetzgebung. Wenn das das Modell ist, das eigentlich zu dem, was wir sehen passt, analytisch, dann sind wir in der Tat in einer Grundsatzverschiebung, die nicht mehr zwingendes Recht als allgemeine Regel aufbaut - womit wir umgehen können, das sind wir gewohnt, da haben wir Argumentationsformen -, sondern sich vom Modell der allgemeinen Regel verabschiedet und das bedeutet natürlich ein Rechtsstaats- und Demokratieproblem, wie Sie es auch gesagt haben. Also ich bin ganz auf Ihrer Seite, würde es aber noch in diesen beiden Richtungen erheblich zuspitzen.

\section{Diederichsen:}

Vielen Dank. Herr Marburger bitte.

\section{Marburger:}

Um an das Letzte von Herrn Rückert kurz anzuknüpfen: Ich glaube nicht, dass wir ein Auslegungsverbot haben. Man darf aber nur so auslegen, wie der Europäische Gerichtshof das macht, richtlinienkonform, alles andere ist weg. Ich wollte aber etwas anderes sagen. Zunächst zum Anfang Ihres Vortrages. Er hat mir sehr gut gefallen. Beim deliktischen Haftungsrecht haben Sie gesagt, der Ausgleichszweck überwiege noch den Präventionszweck. Da stimme ich Ihnen voll und ganz zu. Aber, Frau Wendehorst, es wird nicht mehr lange dauern, dann sind wir beide allein mit dieser Auffassung, denn mehr und mehr tritt die Prävention in den Vordergrund - von vielen Kollegen mit Nachdruck vertreten. Der nächste Punkt: Mir hat Ihre sehr skeptische Haltung gegenüber dem Verbrauchervertragsrecht sehr gut gefallen. Ich will es mal so sagen: Was da alles über uns gekommen ist in den letzten Jahren wird man nicht mehr zurückdrehen können. Wir müssen versuchen, das Beste daraus zu machen. Dahinter steckt - es wurde mehrfach angesprochen - ein bestimmtes Verbraucher-Menschenbild. Es wird immer gesagt in den Erwägungsgründen der Richtlinien, das sei der mündige Verbraucher, der mündige Bürger. Das Gegenteil ist der Fall, es ist der unmündige, der dumme Verbraucher, der zum - und da kommt der Erziehungsgedanke ins Spiel - verantwortungslosen Verbraucher erzogen wird. Und ganz zum Schluss: Die Wirtschaft ist ganz sicher nicht daran interessiert, dass es stärkere Rückgabe- und Rücktrittsrechte im Verbraucherschutzrecht gibt in Europa. Die verkaufen doch über die Grenzen, ohne dass es solche Regelungen gibt und haben es schon immer getan. Und die Grenzbewohner, zu denen ich gehöre, wir haben uns noch nie um die Grenzen gekümmert, wir sind immer dorthin gefahren, wo es am günstigsten ist. Das machen die Luxemburger in Trier und die Trierer in Luxemburg, im nächsten Supermarkt hinter der Grenze. Da spielt irgendeine Grenze und irgendein Verbraucherschutz überhaupt keine Rolle, man guckt, wo der Preis der günstigste ist und die Ware am besten. Dass ich möglicherweise in Luxemburg dann ein Rücktrittsrecht vom Kreditvertrag 
habe, das interessiert mich überhaupt nicht, sondern nur, wie viel Zinsen ich zahlen soll. Also, das ist alles nicht so wild, wie ich es schon gesagt habe. Das Schlimme ist, dass unser gesamtes Zivilrecht aus den Angeln gehoben wird. Schritt für Schritt erleben wir, dass ein Vertrag, der uns früher als bindend galt, heute nur noch ein Vorschlag ist, jedenfalls innerhalb bestimmter Fristen. Und dass an das Rücktrittsrecht - Widerrufsrecht genannt - absolut keine Voraussetzungen geknüpft sind, was früher ja der Fall war.

\section{WENDEHORST:}

Ich versuche kurz zusammenzufassen. Erst einmal Frau Scheiwe und Herr Rückert. Sie haben mich da kalt erwischt. Offen gestanden, ich war so glücklich, endlich ein edukatorisches Element gefunden zu haben, dass ich mich habe hinreißen lassen zu der Bemerkung, es sei eines par excellence. Das ist sicherlich übertrieben. Ich wollte natürlich jetzt nicht in dieses Extrem abgleiten, und werde das auch nicht tun, dass ich sage, alles, was normativ ist, ist zugleich edukatorisch. Ich bleibe bei der Behauptung, dass diese neuen Elemente die Verbraucher zu einem europäischen Verbraucher machen sollen, zu einem Verbraucher, dem bei jeder seiner Aktionen bewusst ist: Würde ich dieses Produkt billiger vielleicht in Dänemark oder in Rumänien bekommen? Das ist meines Erachtens schon eine edukatorische Zielrichtung im engeren Sinne. Aber vielleicht war das ,par excellence“ übertrieben.

Der Binnenmarkt steht im Vordergrund, die Edukation nicht so sehr. Auch das würde ich unterschreiben, selbstverständlich. Der Binnenmarkt ist das Ziel, und die edukatorische Wirkung ist sozusagen schon nur noch das Mittel zum Zweck. Nichtsdestotrotz ist diese edukatorische Zielsetzung da.

Das mit dem référé législatif, das ist wahrscheinlich eine der schwierigsten Fragen, die wir derzeit haben. In der Tat erinnert momentan vieles an Zustände, wie wir sie schon einmal hatten. Aber heute wie damals wird ein référé législatif letztlich an seiner mangelnden Praktikabilität scheitern.

Herr Marburger, Sie haben gesagt: richtlinienkonforme Auslegung. Das ging natürlich alles wunderbar unter Bedingung der Mindestharmonisierung. Da wussten Gerichte, solange sie zugunsten des Verbrauchers judizieren, sind sie auf der sicheren Seite. Unter der Bedingung der Vollharmonisierung ist die richtlinienkonforme Auslegung praktisch durch ein nationales Gericht nicht mehr mit Sicherheit durchzuführen. Man kann nur noch Vermutungen anstellen, wie der EuGH entscheiden würde. Aber strenggenommen müsste man unter der Bedingung der Vollharmonisierung dauernd dem EuGH vorlegen. Vielleicht eine pragmatische Antwort: Das wird fast nur in Deutschland auch tatsächlich so gemacht. In den meisten Mitgliedsstaaten bekümmert man sich aber einfach nicht 
in demselben Maße, und ich fürchte, dass auch die Überlegungen, die dahinter stehen, sind: Man soll die Sache nicht gar so genau anschauen.

Ja, Herr Marburger, Deliktsrecht; ich gebe Ihnen vollkommen Recht, wir haben hier eine dynamische Auslegung. Auch Vorschriften, die ich in meinem Vortrag genannt habe, z.B. dieses Verbraucherlandsprinzip im Kollisionsrecht, haben vom Römischen Schuldvertragsübereinkommen hin zur Rom I-Verordnung eine Umdeutung erfahren: früher Verbraucherschutz, jetzt wieder dieser Binnenmarktgedanke. Genauso ist es mit dem Deliktsrecht. Normen können mit einer Intention A eingeführt worden sein, und irgendwann bekommen sie die Bedeutung B.

Dass die Wirtschaft am Rücktrittsrecht nicht interessiert ist, das habe ich schon bei Christine Langenfeld gesagt, das glaube ich auch. Es sind eigentlich ganz andere Dinge, die tatsächlich die Entscheidungen beeinflussen. Und das macht ja auch die ganze Diskussion um diese Vollharmonisierung so - ich würde fast sagen - verlogen, denn man tut so, als liege das alles daran, ob jetzt die Rücktrittsfrist so oder so lange ist und ob ich jetzt diese oder jene Informationspflicht habe. In Wahrheit sind es doch Dinge wie Sprache und Kultur usw., die hier die grenzüberschreitenden Transaktionen behindern. Da gebe ich Ihnen vollkommen Recht.

DiederichSEN:

Ja, wir haben noch drei Wortmeldungen. Herr Simon, Herr Zimmermann und Herr Eichenhofer bitte.

SimOn:

Was Sie schilderten, dass nämlich Teile des klassischen Vertragsrechts durch Regulierungsnormen von innen heraus ausgehöhlt bzw. überlagert werden, ist ein Vorgang, den man eigentlich schon seit 120 Jahren beobachten kann. Denn Vergleichbares war ja im ausgehenden 19. Jahrhundert, zuerst vielleicht beim Versicherungsvertrag, dann beim Arbeitsvertrag und dann im Mietrecht, auch der Fall: Klassisches Vertragsrecht wird ausgehöhlt durch eine Flut von Regulierungsnormen, die vom Gesetzgeber erlassen werden. Und es ist dieses typische Amalgam aus öffentlich-rechtlichen Rahmennormen und zwingendem Vertragsrecht, was dabei entsteht. Die Konsequenz war, dass sich ein eigenständiges Arbeitsrecht und ein eigenständiges Versicherungsrecht gebildet hat. Was Sie beschreiben, ist jetzt eine neue Vertragskonstellation, eine bestimmte Form eines Kaufvertrages, sodass sich im Laufe der Zeit höchstwahrscheinlich hier eine eigene Dogmatik ausbilden wird, die aber vielleicht doch auch dazu beitragen wird, durch die Ausbildung eines dogmatischen Gerüstes dieses Sonderprivatrecht dann wieder zurückzubinden an die allgemeine Dogmatik. So war es ja in gewisser Weise im Arbeits- und Mietrecht. Vielleicht muss man es gar nicht so 
dramatisch sehen, wenn man schaut, wie das in anderen Bereichen ausgegangen ist.

\section{DIEDERICHSEN:}

Herr Zimmermann bitte.

\section{ZiMMERMANN:}

Anknüpfend an das, was Herr Simon gerade gesagt hat, möchte ich einen kleinen Gegenakzent setzen. Man sollte nicht Europa für alles blamieren, was hier so passiert. Welches sind die Mechanismen, die wir für den Verbraucherschutz haben? Es sind vier typische Mechanismen: Informationspflichten, Widerrufsrechte, AGB-Kontrolle und zwingendes Recht. Alles oder fast alles von dem hatten wir schon im deutschen Recht, bevor das europäische Recht über uns gekommen ist. Der BGH hat völlig autonom in den 1970er und 1980er Jahren Informationspflichten stark ausgeweitet. Die AGB-Kontrolle ist knapp 20 Jahre älter im deutschen Recht als die Klausel-Richtlinie. Zwingendes Recht hat es immer gegeben in gewissem Rahmen im BGB. Auch da hat es schon eine Verstärkung gegeben in den 1970er, 1980er Jahren. In gewisser Weise neu ist vielleicht das Widerrufsrecht, obwohl das Haustürwiderrufsrecht vom BGBGesetzgeber eingeführt worden ist, bevor die Haustürwiderrufsrichtlinie erlassen worden ist. Und es gab auch Tendenzen, stärker Lösungsrechte zu gewähren, nicht geradezu Reurechte, aber doch immerhin Lösungsrechte, Stichwort culpa in contrahendo usw. Das Ganze ist eine Tendenz, die Herr Canaris in seinem Zivilrechtslehrervortrag als „Materialisierung des Zivilrechts“ bezeichnet hat. Das ist also etwas, was schon das deutsche Recht geprägt hat, jetzt aber durch das Richtlinienrecht sehr verstärkt worden ist. Man kann sich fragen und man muss sich fragen, ob das alles sachgerecht ist. $\mathrm{Ob}$ also etwa dieses viele neue zwingende Recht, das eingeführt worden ist, erforderlich ist. Warum müssen alle Regeln über den Verbrauchsgüterkauf zwingend gestellt sein? Warum muss das Pauschalreiserecht zwingend sein? Das ist das Thema der acquis-Revision, der Revision des verbrauchervertragsrechtlichen acquis communautaire. Das hat die Europäische Kommission sich seit einer Mitteilung aus dem Jahr 2001 auf die Fahnen geschrieben. Eine acquis-Revision, die diesen Namen verdient, ist aber bis heute nicht durchgeführt worden, weder vom europäischen Gesetzgeber noch von der Wissenschaft. Die acquis-Gruppe hat das nicht gemacht, andere auch nicht. Da müssen wir uns auch ein bisschen an die eigene Nase fassen. Wir haben Vorschläge für solch eine acquis-Revision bisher in systematischer und überzeugender Form nicht vorgelegt. Das ist, glaube ich, eine ganz dringende Aufgabe: zu prüfen, was von diesem Verbraucher-acquis, der sich entwickelt hat, überhaupt rational begründbar ist. Also etwa: Erfüllen die Regeln, die es gibt, überhaupt den Zweck, den sie erfüllen sollen? Vieles ist nämlich dysfunktional. 
Das wäre grundlegend aufzuarbeiten. Wenn man das tut, dann glaube ich aber wäre es geradezu gefährlich für die Integrität des Privatrechts, wenn man dieser Zwei-Reiche-Lehre folgt. Wir hätten dann auf der einen Seite das allgemeine Vertragsrecht und auf der anderen Seite das Verbrauchervertragsrecht. Und dann wäre also das allgemeine Vertragsrecht das Reich der unbegrenzten Vertragsfreiheit. (Ich meine aber, spätestens seit Frau Hofer wissen wir: Auch im 19. Jahrhundert war die Vertragsfreiheit nicht unbegrenzt und rein formal. Es ist von Anfang an eine materialisierte Vertragsfreiheit.) Also: Wir hätten auf der einen Seite die Vertragsfreiheit und auf der anderen Seite ein soziales Recht zum Schutz des Verbrauchers oder ein regulatorisches Recht. Vertragsfreiheit ist nun aber kein Wert an sich. Vertragsfreiheit ist Mittel zur Verwirklichung der Selbstbestimmung. Aber es ist beim Vertragsrecht immer Mittel zur Verwirklichung der Selbstbestimmung zweier Parteien. Wir haben eben typische Situationen, in denen nicht beide Parteien eine selbstbestimmte Entscheidung treffen können. Das lässt sich an vielen Beispielen zeigen, z.B. beim Haustürwiderrufsrecht. Ich glaube, dass sich dort ein Widerrufsrecht begründen lässt: Stichwort Überrumpelung. Das lässt sich ohne Weiteres in das Modell unseres Vertragsrechtes einfügen, wenn man nicht auf einer formalistischen Definition der Vertragsfreiheit besteht. Und deshalb denke ich, ein gereinigter und revidierter acquis lässt sich durchaus einfügen und muss eingefügt werden in das allgemeine Vertragsrecht. Ich halte es für falsch, die beiden Bereiche so strikt voneinander zu trennen.

DiEDERICHSEN:

Vielen Dank! Und nun Herr Eichenhofer als Letzter, bitte.

\section{EICHENHOFER:}

Ich wollte noch Aufklärung darüber haben, was edukatorisch ist bei den Phänomenen, die Sie uns beschrieben haben. Sie können entweder sagen, es sind die Inhalte. Der Verbraucherschutz als solcher hat eine edukatorische Komponente. Edukatorisch kann auch die Form sein - also eine Charta der Verbraucherrechte! Ich kenne eine Charta der Patientenrechte. Zu ihr kann man sagen: ,Ja, das ist eine neue Rechtsform. "Ich würde aber sagen, es ist nicht in erster Linie eine edukatorische, sondern eine archaische Rechtsform, weil es sich um leges imperfectae handelt: Man stellt eine Reihe von Prinzipien auf, wie wir uns das Verhältnis von Unternehmer und Verbraucher denken, sagen aber nicht: Was folgt, wenn eines dieser Prinzipien nicht beachtet wird? Also insoweit ist der Versuch, auf europäischer Ebene etwas durch Charta-Bestimmungen zu fixieren, was man durch Rechtsbestimmungen aus den verschiedensten Gründen nicht festhalten kann, im Grunde genommen ein unzulänglicher, archaischer, in der Tradition des 18. Jahrhunderts stehender Versuch. Ich frage mich aber, ob das nicht auch eine Folge eines Kollisionsrechts ist, das auf dem Günstigkeitsprinzip beruht. 
Das Verbraucherkollisionsrecht beruht auf dem Günstigkeitsprinzip wie auch das internationale Arbeitsrecht. Und die Folge dieses Kollisionsrechts ist, dass im konkreten Einzelfall ein Recht zur Anwendung kommt, das in keinem einzigen Land so gilt, weil es sich eben aus mehreren Rechten zusammensetzt, also fiktionales Recht ist, das einzig aus kollisionsrechtlichen Gründen auf einen Fall zur Anwendung kommt. Das heißt mit anderen Worten, wir haben es nicht mit 30 Privatrechten zu tun, sondern - wenn wir das Günstigkeitsprinzip zu Ende denken - mit ungleich mehr. Und in einem solchen Kollisionsrecht, in dem sich auch die Beurteilungsmaßstäbe dem nationalen Rahmen vollständig entziehen, werden virtuelle Beurteilungsgrundlagen geschaffen. Dafür ist das Bedürfnis nach Reduktion der Komplexität riesengroß. Und die Charta befriedigt dieses Bedürfnis - aber auf eine unvollkommene Weise.

\section{WENDEHORST:}

Ich beantworte die Fragen in umgekehrter Reihenfolge. Dass die Charta auf unvollkommene Weise befriedigt, da gebe ich Ihnen vollkommen Recht. Sie haben sich auf das bezogen, was ich über Kommissarin KunewA gesagt habe. KunEwA schwebt aber natürlich schon eine Charta vor, die sich auf eine Verordnung oder auf vollharmonisiertes Verbraucherrecht stützt, d.h. eine Charta, die dem Individuum seine Rechte noch einmal deutlich macht, weil das Individuum, was wohl zutrifft, durch die Lektüre von Rechtsnormen und Reflexionen des Verbraucherlandsprinzips zu diesem Schluss einfach kommt. Ansonsten gebe ich Ihnen vollkommen Recht, dass das ein unvollkommenes Steuerungselement ist.

Ob die Form oder der Inhalt edukatorisch ist? In diesem Fall vermutlich in der Tat die Form. Bei anderen Dingen, z.B. in den europäischen Formblättern, bin ich mir nicht so ganz sicher, ob man Form und Inhalte trennen kann. Das will ich jetzt lieber nicht versuchen.

Aber jetzt zu den noch schwierigeren Fragen, ob es sich um ein bekanntes Phänomen handelt, wie wir damit umgehen können und wie das mit der ZweiBereiche-Lehre ist. Zunächst einmal möchte ich klarstellen, dass ich sicherlich keine Anti-Europäerin bin, ich glaube als die bin ich auch noch nie irgendwo in Erscheinung getreten. Also Europa für alles zu blamieren ist sozusagen nicht meine Intention, und dass vieles, was in Europa passiert, den Weg über Deutschland nimmt, das ist auch allgemein bekannt. Z.B. sind Strukturen, die wir in verbrauchervertragsrechtlichen Richtlinien finden, vielfach vom deutschen Recht abgeschrieben. Bei der Haustürgeschäftegeschichte war das so eine Wechselwirkung: Man hat in Europa Einfluss genommen, dann wusste man, wann es kommt, und dann hat man vorweggenommen umgesetzt. Zustimmen möchte ich auch, dass wir dringend eine acquis-Revision brauchen. 
$\mathrm{Ob}$ die Zwei-Bereiche-Lehre falsch ist, da muss ich sagen, dass kommt eben in meinen Augen darauf an, was diese acquis-Revision bringt. Wenn die acquisRevision es schafft, das Verbrauchervertragsrecht und das europäische Verbrauchervertragsrecht kompatibel zu gestalten, so dass ich vielleicht wieder eine Feinsteuerung der Prinzipien habe, so dass ich vielleicht wieder auf herkömmliche methodische Grundsätze und Argumentformen zurückgreifen kann, dann lasse ich mir das mit der Zusammenführung beider Bereiche schon gefallen. Es ist dann allenfalls eine pragmatische Frage, ob man das regelungstechnisch kombinieren sollte oder ob nicht die Frequenz, mit welcher der europäische Gesetzgeber die Dinge wieder ändert, dafür spricht, das Verbraucherrecht vielleicht doch auszulagern. Wenn es allerdings nicht gelingt, die acquis-Revision entsprechend durchzuführen, wenn also das europäische Verbrauchervertragsrecht so bleibt, wie es derzeit ist, dann, muss ich gestehen, bin ich entschieden gegen eine Zusammenführung beider Rechtsmaterien. Wenn man sich z.B. jetzt die Umsetzung der Verbraucherkreditrichtlinie ansieht im BGB, das ist eine regelungstechnische Katastrophe. Dass erst Monate nach Erlass des Gesetzes die himmelschreiendsten Richtlinienwidrigkeiten erkannt und wieder korrigiert wurden, das spricht ja für sich. Das ist so kompliziert, dass man das auch im Justizministerium gar nicht mehr selber durchschaut. Wenn es nicht gelingt den acquis kompatibel zu gestalten, dann bin ich sehr skeptisch, was die Zusammenführung beider Bereiche anbelangt.

Dass es sich um ein altes Phänomen handelt, ja und nein. Erst einmal, dass es schon vergleichbare Phänomene gegeben hat, hindert natürlich nicht daran, auf generell unterbewertete Konflikte, die wir jetzt in unserer Rechtsordnung haben, hinzuweisen. Zweitens denke ich, ist das einfach eine Frage, wie weit das Privatrecht langsam erodiert und wie viel noch übrigbleibt. Sicherlich, es waren schon ganz wichtige und zentrale Bereiche, die abgespalten worden sind. Aber es blieb immer noch genug übrig. Wenn wir jetzt an Dinge gehen wie z.B. das Verbrauchsgüterkaufrecht oder die AGB-Kontrolle, da fragt man sich dann, insbesondere im Kaufrecht: Was bleibt eigentlich noch übrig? Also ich fürchte, dass wir langsam vor einen Punkt geraten, wo fast nichts mehr übrigbleibt, und deswegen würde ich hier doch einen qualitativen Sprung sehen.

\section{DiEDERICHSEN:}

Dankeschön. Ja, wir haben etwas mehr als eine Viertelstunde überzogen, aber ich meine, das sollte uns das BGB wert sein. 